\section{Brightfield Illumination of Large Field Sizes}

Theodore M. Clarke

Metallurgical Failure Analysis Consultant tclarke@ligtel.com

Microscopists and scientific photographers sometimes find it desirable or necessary to record a larger field size than the lowest power objective can cover using brightfield illumination. This applies to the largest thin sections examined by biologists and geologists in transmitted light and the opaque, planar specimens examined by the materials scientist in reflected light. These needs were once well met by Leitz with their Panphot and Aristophot systems and to a lesser extent by Zeiss with their Ultraphot II. Sadly,

Double Condanser Mothed

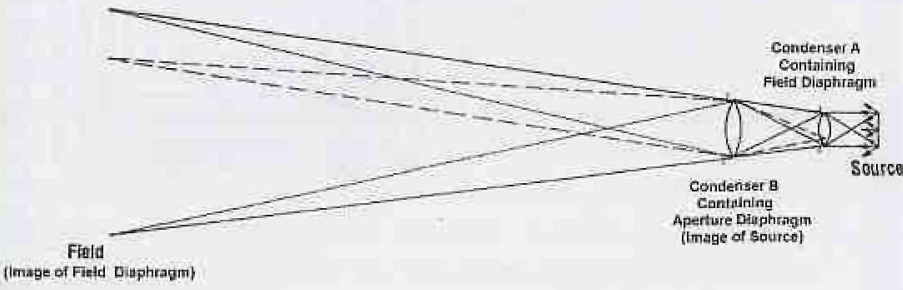

this capability has been lost because this type of equipment is no longer manufactured. Most users of the microscope are unaware of its operating principles and the different illuminating methods, so they are unaware of the benefits once provided by these older imaging systems. Henry Clifton Sorby, the father of metallography and petrography, was the first to recognize the on the flat specimen is either transmitted or reflected into the objective lens, depending on whether transmitted through a thin, transparent specimen or reflected by the mirror-like surface of a metallographic specimen. This is a review of an earlier article: Clarke, T. M. "Brightfield Illumination of Complete Metallographic Specimens": The Microscope 1996, 44:2, 59-80. The details of the design and construction of two gross specimen illuminating systems are presented along with photographic examples. The larger illuminator is capable of illuminating a field size of 82 by $106 \mathrm{~mm}$ in transmitted light and 68 by $89 \mathrm{~mm}$ in reflected light with an imaged field diaphragm and the illumination aperture diaphragm imaged in the aperture of the macro lens. A fiber-optic light guide is used as the light source of a double condenser illuminator used in combination with a field lens near the specimen. The graphical design method uses high-school optics, and aperture size is determined by the Abbe resolution criterion for useful magnification.

Scientific photographers may be acquainted with the use of a Nicholas illuminator to obtain even illumination for photomacrography. The Nicholas illuminators use the double condenser principle. The commercially available Nicholas illuminators usually suffer from low light intensity, and they have their elements in fixed positions without an aperture diaphragm to control the illumination contrast (numerical aperture). The operating principle of the double condenser illuminator is illustrated in Figure 1. Figure 2 shows a double condenser fiber-optic illuminator made from an Olympus $35 \mathrm{~mm}$ camera bellows fitted with a $13 \mathrm{~mm} f 11.8$ movie camera lens and a $50 \mathrm{~mm}$ $\mathrm{f} / 1.835 \mathrm{~mm}$ camera lens. The obsolete movie camera lens and light-guide end, with a $1 / 4^{n}$ bundle size, fit in an adapter replacing the camera-mounting ring shown together in Figure 3 . The sleeved end of the light guide slides in the bore of the adapter with a thumbscrew to lock the position of the light-guide end (the light source in Figure 1). The $50 \mathrm{~mm}$ f/1.8 camera lens is replaced with a $28 \mathrm{~mm}$ f/2.8 camera lens when this illuminator is used with the larger field size illuminator. Figure 4 shows this illuminator used
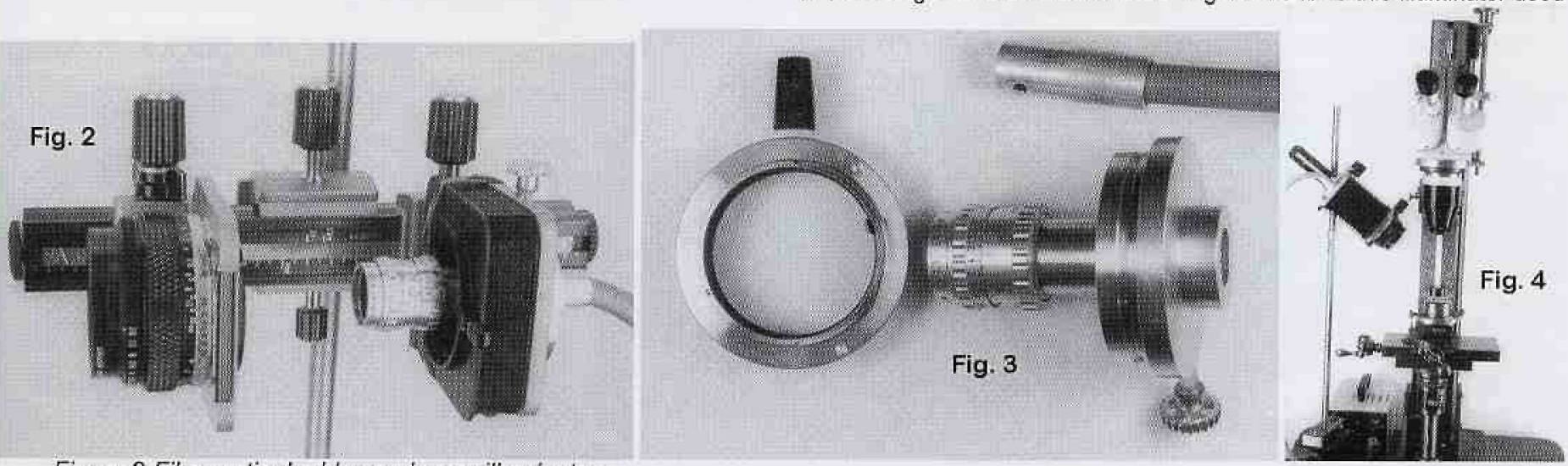

Figure 2-Fiber optic double condenser illuminator.

Figure 3-Fiber optic light guide housing with an attached movie camera lens.

Figure 4-Darkfield illumination with a double condenser illuminator.

important differences between reflected brightfield and reflected darkfield illumination when viewing his opaque metallographic specimens. His first metallographic images were of large sections at low power. His reflected brightfield illumination for the gross sections was obtained with a reflector below the objective, a feature retained in the systems I will describe later Brightfield illumination means that the cone of light illuminating each point to provide darkfield illumination of a metallographic specimen viewed with a stereo microscope, which can be interchanged with a bellows mounted camera for imaging. Figure 5 shows a complete metallographic specimen cut from a fillet weld of low carbon steel to medium carbon steel, which was prepared in a $11 / 2$ " metallurgical mount of glass fiber reinforced, black plastic resin and photographed with darkfield illumination by the double
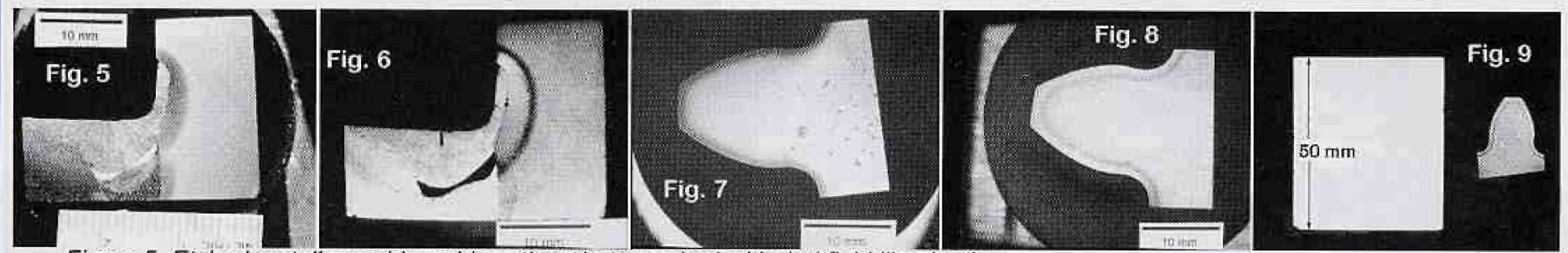

Figure 5-Etched metallographic weld section photographed with darkfield illumination.

Figure 6-Same metallographic weld section as in Figure 5 except photographed with brightfield illumination.

Figure 7-Etched gear tooth metallographic section photographed with darkfield illumination.

Figure 8-Same metallographic section of gear tooth shown in Figure 7 except photographed with brightfield illumination. 


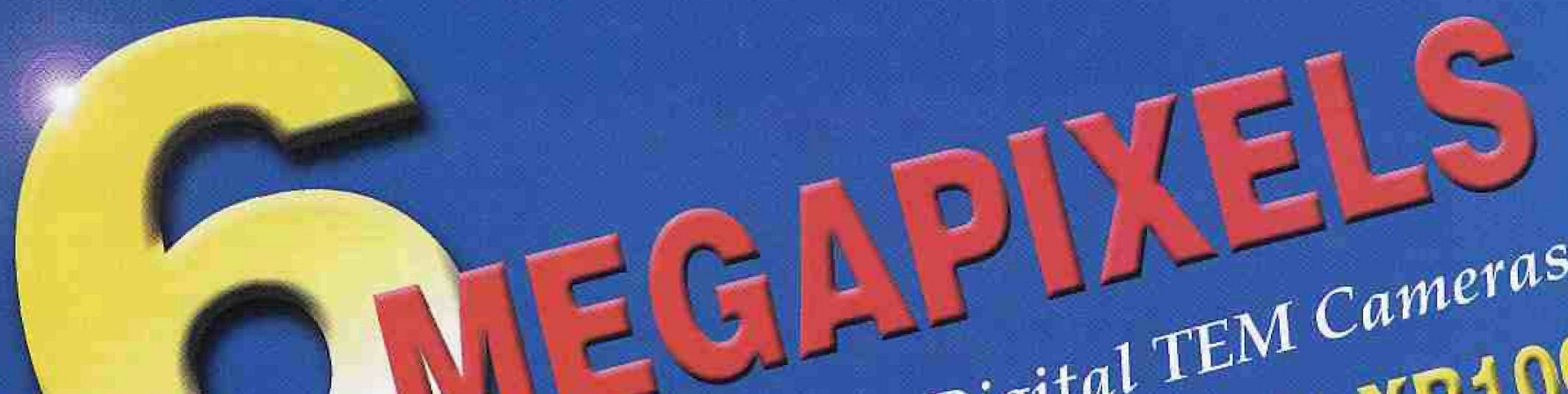
High Definition

TEM
NTIECRATION AMT's $\times$ : 60

\&. $\times 100$

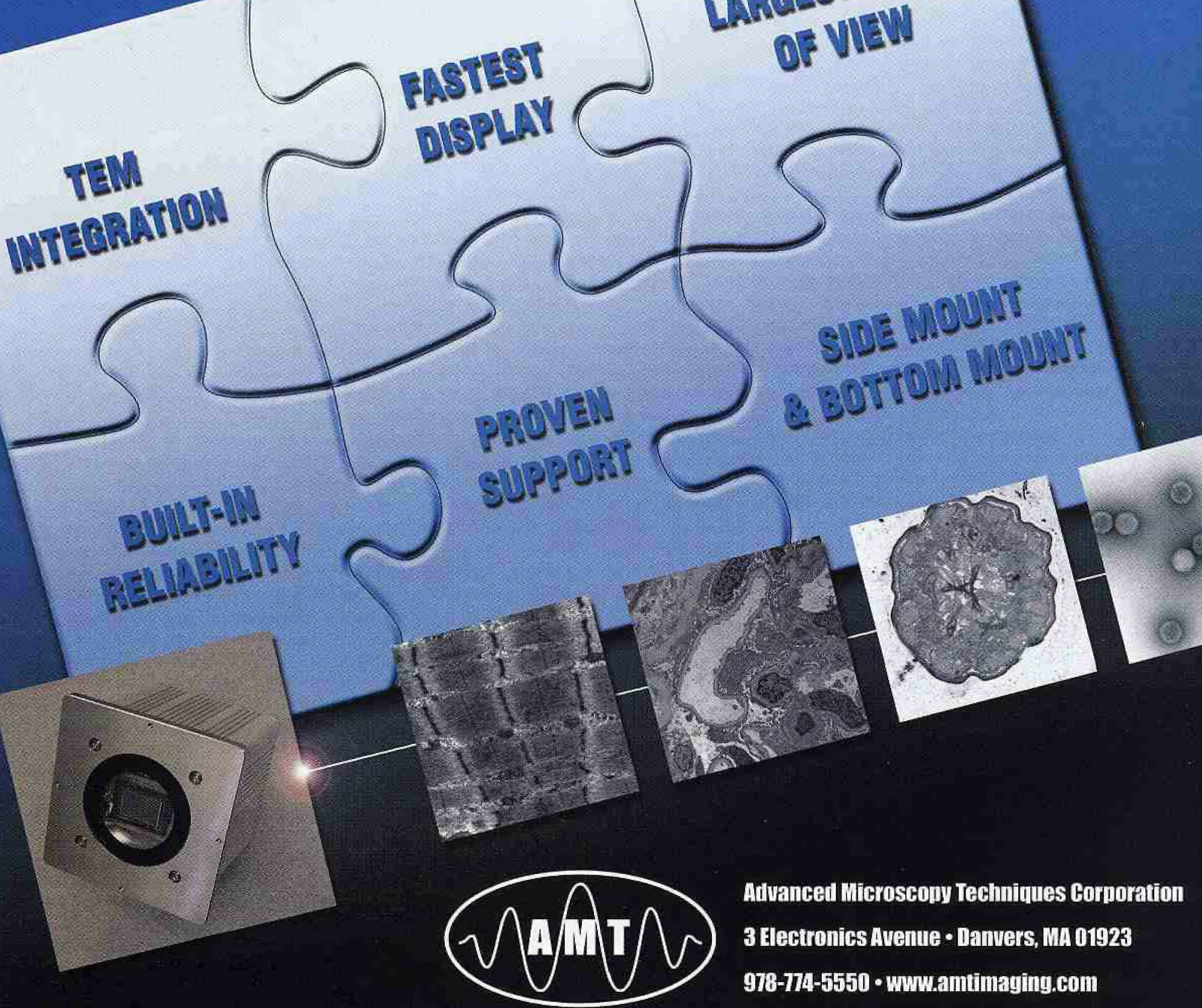



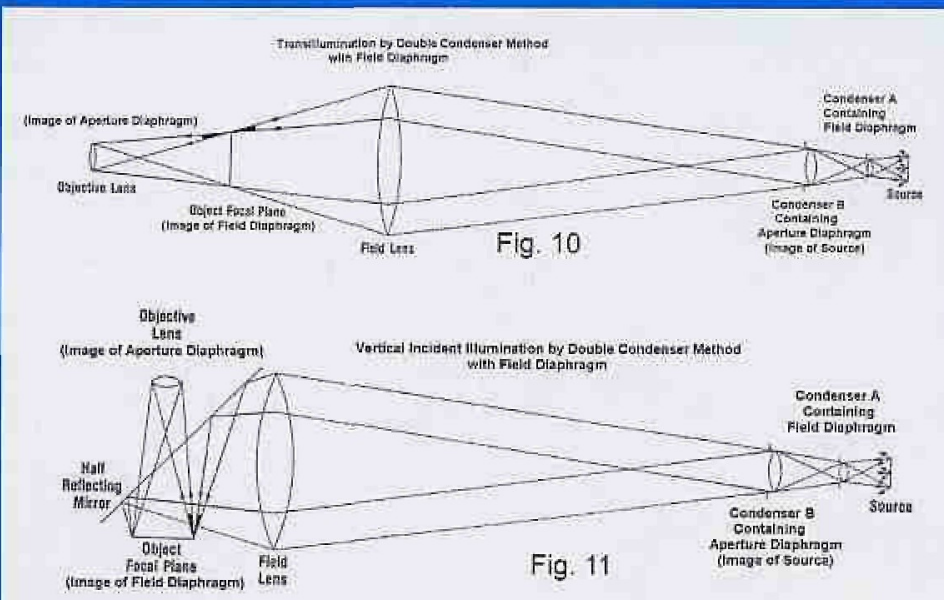

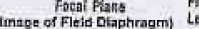

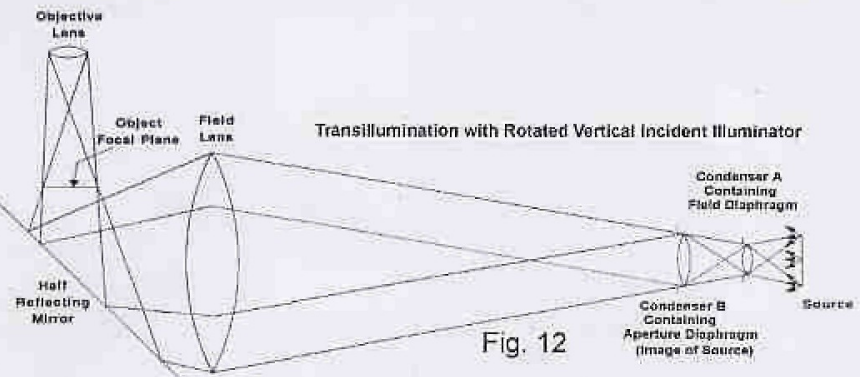

condenser shown in Figure 4. Figure 6 shows the same specimen imaged with brightfield Illumination using the smaller field size brightfield illuminator described later. Henry Clifton Sorby would have understood the cause of the difference in appearance of the same field. He would know that the black plastic absorbs the incident light and would remain black whether with brightfield or darkfield illumination. He also knew that the high-hardness heat-affected zone in the medium carbon steel would not be significantly etched, and therefore, would specularly reflect the brightfield illumination appearing bright in the brightfield image and dark in the darkfield image. You can now realize the confusion that can be caused when the higher magnification images recorded with the microscope using Koehler illumination are compared with the same regions on the gross specimen typically recorded with darkfield illumination. Figures 7 and 8 show a complete metallographic section of a tooth sectioned from a carburized and hardened gear. The high-hardness outer layer on the tooth shows the same image contrast reversal seen with the high-hardness heat-affected zone in the weldment specimen. Figure 9 shows the same gear tooth specimen recorded with reflected brightfield illumination along with a $50 \mathrm{~mm}$ wide mirror in the much larger field covered by the larger illuminator.

The operating principle of the brightfield illuminators is illustrated with the ray diagram of Figure 10 for transmitted brightfield. The distance from the field lens to the specimen is excessive for transmitted brightfield, but was drawn this way to permit folding the illuminating light path ninety degrees by adding the beam splitter necessary for reflected brightfield illumination shown in the ray diagram of Figure 11 . The beam splitter can be a thin sheet of glass, but a more efficient beam splitter has a partially silvered coating and an anti-reflection coating on the opposite side, to avoid ghost images. Typical of previous gross specimen illuminators, the light source with its aperture diaphragm is imaged in the aperture of the recording macro lens. The ray diagrams are drawn based upon calculation of conjugate positions and magnifications using the simple lens equations. The field diaphragm, located in the short focal length lens closest to the light guide end in the double condenser illuminator, is imaged on the object focal plane by the combination of the front lens of the double condenser and the large diameter field lens. The magnification of the image of the field diaphragm and distance to it from the front lens of the double condenser are calculated from the simple lens equations by using the image formed by the first condenser lens as the object for the second lens, while observing the correct sign conventions. The necessary illumination aperture size, controlled by the diaphragm in

the lens closest to the field lens, is calculated based upon the final printed image magnification. The numerical aperture can be calculated from the tangent of one half the included angle between the camera lens aperture diameter and the distance to the object focal plane. I recommend a more stringent Abbe criterion for image quality. The final magnification should not exceed 500 times the numerical aperture of the recording camera lens. The camera lens maximum aperture will normally greatly exceed the value calculated from the Abbe criterion, but it is convenient to leave the camera lens diaphragm opened fully without affecting the brightfield contrast. The reflected light illuminators can be used in transmitted mode as shown by the ray diagram in Figure 12.

The work involved in building a brightfield illumination system for gross specimens, especially one with a beam splitter for reflected brightfield, is simplified if a vertical illuminator is commercially available that is suitable for the desired field size. At the time the work was done for my 1996 article, a vertical illuminator PM-EL 80 was available from Olympus for their $80 \mathrm{~mm}$ macro lens. This illuminator is intended to be mounted to the front of the macro lens but can illuminate a much larger field size by being held just

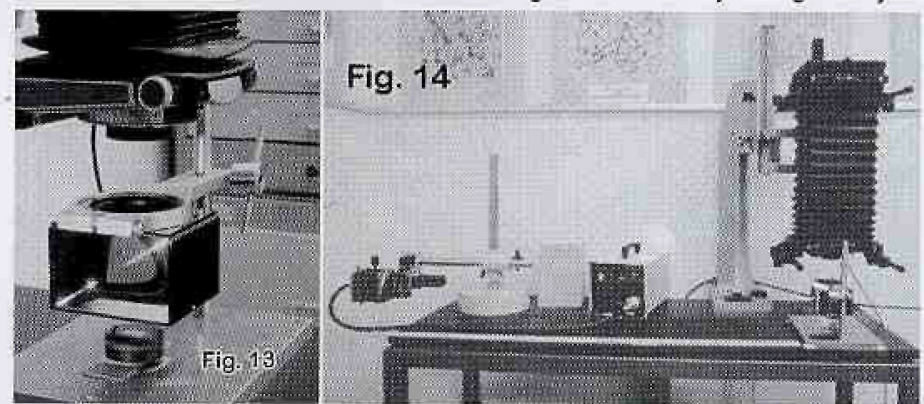

Figure 13-OlympuS PM-EL80 vertical illuminator mounted in a stand above a metallographic specimen.

Figure 14-View camera system using the Olympus PM-EL80 vertical illuminator for reflected brightfield

above the specimen as shown in Figure 13. The focal length of its Fresnel field lens is $180 \mathrm{~mm}$. An illuminating system using this Olympus vertical Illuminator was used to obtain the brightfield images shown in Figures 6 \& 8. These images were recorded on $4 \times 5$ in. Polaroid sheet film using the bench mounted view camera system shown in Figure 14. Since aligning the illuminating system is time consuming, this system is normally left already setup for the next job in the $\mathrm{CNH}$ metallography laboratory. This illumination system can also be used with a digital or $35 \mathrm{~mm}$ camera focused on the same field size with its lens at the same position as the view camera lens.

Occasionally there was need to record a much larger field size in reflected brightfield illumination. This led to the construction of the vertical

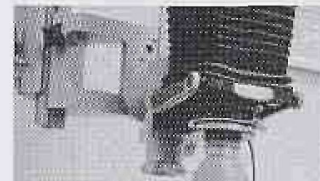

Fig. 15

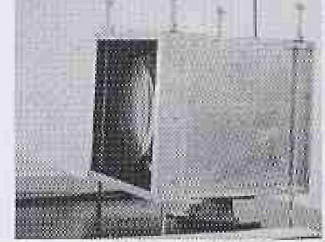

Figure 15-Homebuilt large field vertical illuminator.

Figure 16-View camera system for reflected brightfield Illumination using the large field vertical illuminator.

illuminator shown in Figure 15 for the imaging system shown in Figure 16. The $152 \mathrm{~mm}$ diameter $300 \mathrm{~mm}$ focal length polished glass field lens and the $127 \times 178 \mathrm{~mm}$ metallic coated beam splitter (half mirror) were obtained from Edmund Industrial Optics for this mirror housing built in my home machine shop. This beam splitter has an antireflection coating on the side opposite the metallic coated side to prevent ghost images from multiple reflections. 


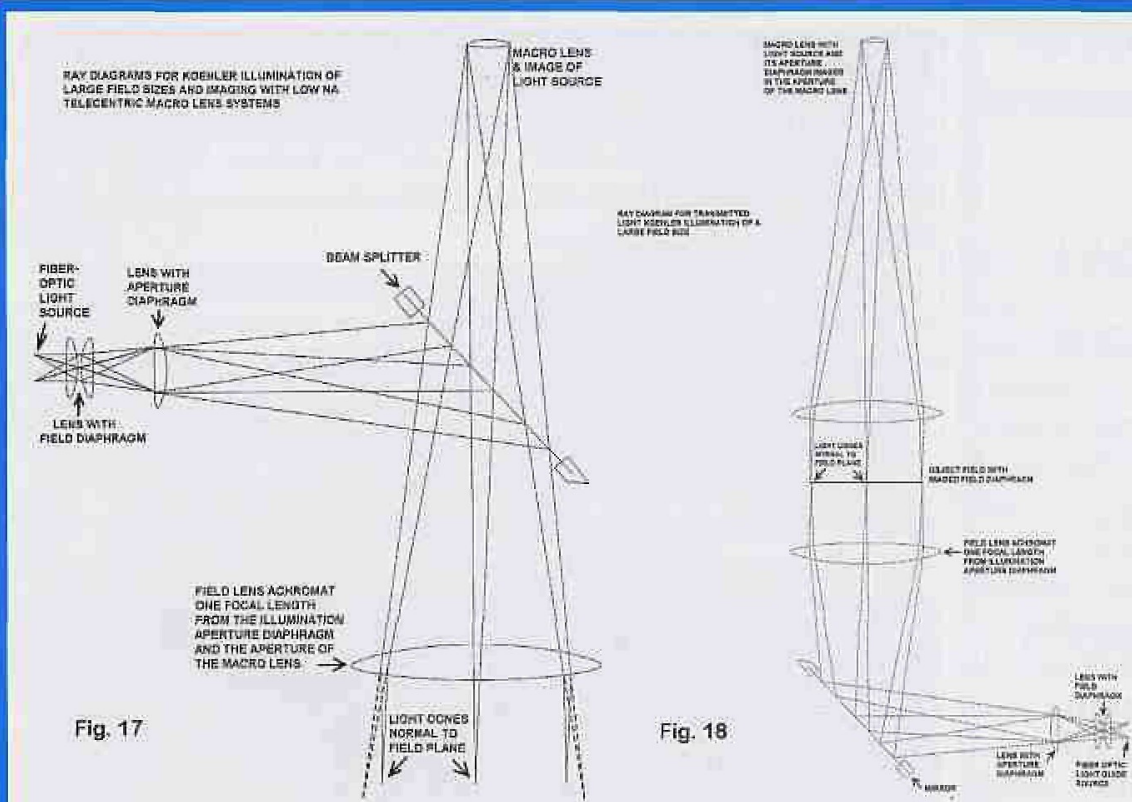

illuminator is capable of brightfield illumination of a complete $4 \times 5$ in. negative in transmitted brightfield illumination.

The illumination system shown in Figure 14 is too large to be suitable in some laboratories. A shorter focal length field lens would help. Making the system suitable only for transmitted light with the field lens adjacent to the object focal plane, as done with the Leitz Aristophot, would yield the most compact system. The most compact arrangement for reflected brightfield illumination of large field sizes is to place the field lens in the imaging system very close to the abject focal plane. The field lens then becomes a part of a telecentric imaging system shown in the ray diagram of Figure 17 . The field lens must then be an achromat with a very efficient multilayer anti-reflection coating to prevent visible reflections from the lens surfaces in the images. I have tested such a system using the salvaged front elements of a $135 \mathrm{~mm}$ telephoto lens as the field lens, but there was a reflection from the field lens visible in the images. Since large diameter achromats with the necessary broadband anti-reflection coating are not affordable for a home experimenter, I have decided the next system will use what Zeiss calls the Antiflex system

The large field size means that this system operates at a very low numerical aperture using a $28 \mathrm{~mm}$ focal length $\mathrm{f} / 2.8$ camera lens on the front of the double condenser illuminator. I found that Fresnel lenses showed artifacts on a first-surface mirror test object located at the object focal plane when testing the alignment of the illuminating system. The system using the Olympus PM-EL80 vertical illuminator does not exhibit artifacts from the Fresnel field lens unless the illumination aperture is reduced well below the value needed to satisfy the Abbe criterion. Figure 9 demonstrates the performance of this illuminator in reflected light with a first surface mirror along side a $11 / 2$ metallographic specimen at the object focal plane. This used in stereo microscopes with coaxial reflected brightfield illumination to avoid the lens reflection artifacts. This system will also have the same field size capability in transmitted brightfield, but this will require an additional field lens just below the object focal plane as shown in Figure 18. This type of illumination for large specimens is the low magnification equivalent to Koehler illumination, with illuminating and image forming cones of light normal to the object focal plane.

Reference:

Smith, Cyril Stanley, A History of Metallography: The Univ. of Chicago Press, 1960.

\section{If you have a microscopy application...}
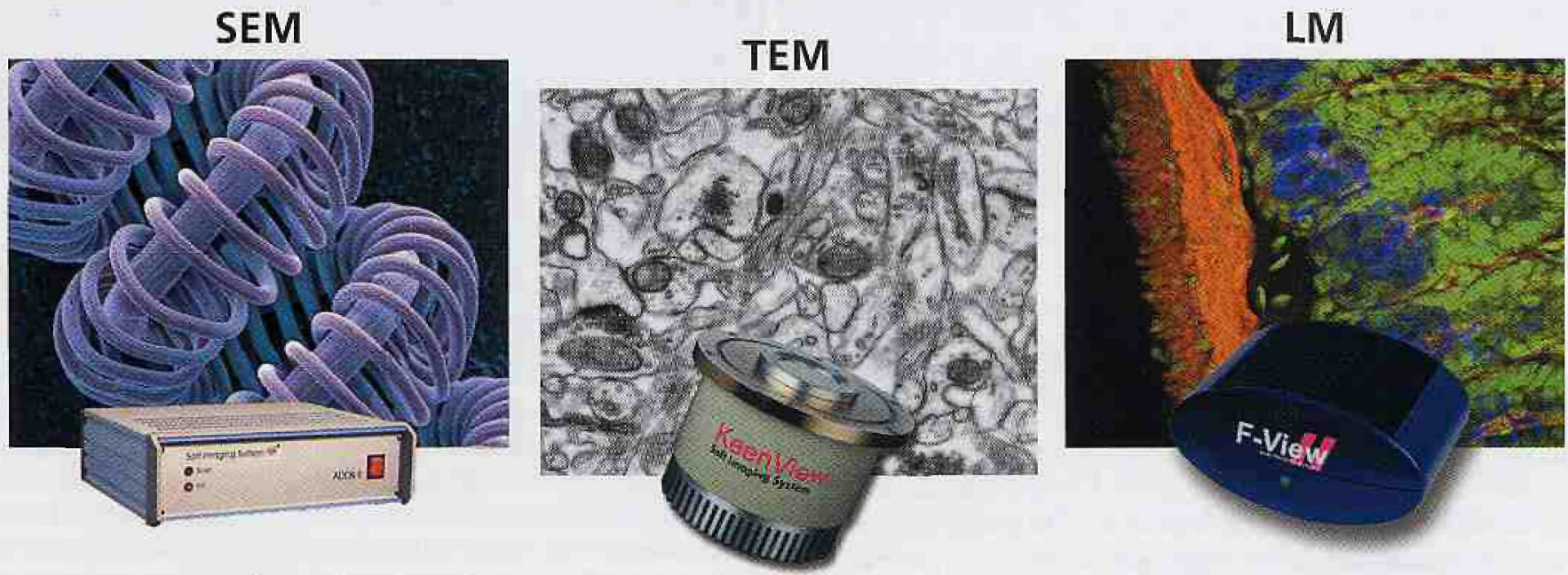

Soft Imaging System offers high resolution, high sensitivity, easy to install cameras for Transmission Electron Microscopes, and Light Microscopes. A digital beam interface is offered for Scanning Electron Microscopes. All of our hardware systenns integrate with analysi5 ${ }^{\circ}$ software - an image analytical system that provides you with microscope control, camera operation, optimized image accuisition, manual or automated image analysis, database archiving, report generation and more.

... we have your solution.

Digital Solutions for Imaging and Microscopy Soft Imaging System 